\title{
Inhalt.
}

\section{Allgemeiner Teil.}

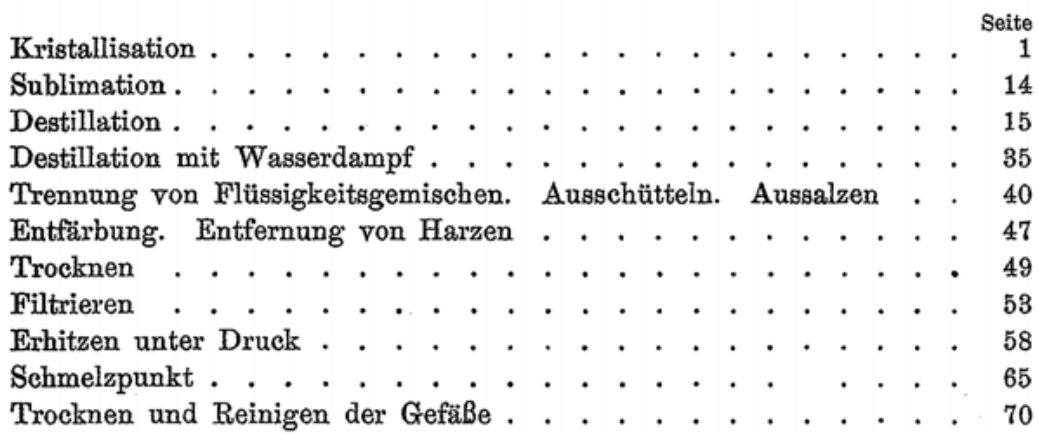

\section{Organisch-analytische Methoden.}

Qualitativer Nachweis des Kohlenstoffs, Wasserstoffs, Stickstoffs,

Schwefels und der Halogene . . . . . . . . . . . . . 72

Quantitative Bestimmung der Halogene nach CARIUs. . . . . . . 75

Quantitative Bestimmung des Schwefels nach CARTUS . . . . . . 81

Quantitative Bestimmung des Stickstoffs nach Dumas . . . . . . $8 \check{0}$

Quantitative Bestimmung von $\mathrm{C}$ und $\mathrm{H}$ nach LreBIG . . . . . . 96

\section{Spezieller Teil.}

\section{Aliphatisehe Reihe.}

1. Reaktion: Ersatz alkoholischer Hydroxylgruppen durch Halogen . 109

2. Reaktion: Darstellung eines Säurechlorides aus der Säure. . . . 118

3. Reaktion: Darstellung eines Säureanhydrides aus dem Säurechlorid und dem Natriumsalz der Säure. . . . . . . . . . . . 124

4. Reaktion: Darstellung eines Säureamides aus dem Ammoniumsalz der Süure . . . . . . . . . . . . . . . . . . . . 128

5. Reaktion: Darstellung eines Säurenitriles aus einem Säureamid. . 131 
6. Reaktion: Darstellung eines Säureesters aus Săure und Alkohol 134

7. Reaktion: Substitution von Wasserstoff durch Chlor . . . . . 140

8. Reaktion: Oxydation eines primären Alkohols zu einem Aldehyd. 144

9. Reaktion: Darstellung eines primären Amins aus dem Säureamid der näcbst höheren Reihe . . . . . . . . . . . . . . 151

10. Reaktion: Synthese von Ketonsäureestern bezw. Polyketonen mit Hilfe von Natrium bezw. Natriumalkoholat. . . . . . . . 155

11. Reaktion: Synthese der homologen Essigsäuren mit Hilfe von Malonsăureester . . . . . . . . . . . . . . . . . . . . 160

12. Reaktion: Darstellung eines Kohlenwasserstoffs der Äthylenreihe durch Abspaltung von Wasser aus einem Alkohol. Vereinigung mit Brom . . . . . . . . . . . . . . . . . . . . 166

13. Reaktion: Ersatz von Halogen durch alkoholische Hydroxylgruppen 171 Utbergang aus der aliphatischen Reihe in die aromatisehe.

Dimethylcyklohexenon bezw. s-Xylenol sus Äthylidenbisacetessigester (RingschlieBung bei einem 1.5 Diketon nach KNoevenages) . . 175

\section{Aromatische Reihe.}

1. Reaktion: Nitrierung eines Kohlenwasserstoffs . . . . . . . . 183

2. Reaktion: Reduktion eines Nitrokörpers zu einem Amin . . . . 187

3. Reaktion: a) Reduktion eines Nitrokörpers zu einem Hydroxylaminderivat. b) Oxydation eines Hydroxylaminderivates zu einem

Nitrosokörper
4. Reaktion: Reduktion eines Nitrokörpers zu einem Azoxy-, Azo- bezw. Hydrazokörper . . . . . . . . . . . . . . . . . . 197

5. Reaktion: Darstellung eines Thioharnstoffs bezw. eines Senföles aus Schwefelkohlenstoff und einem primären Amin . . . . . . 203

6. Reaktion: Sulfurierung eines Amins . . . . . . . . . . 206

7. Reaktion: Ersatz der Amido- bezw. Diazogruppe durch Wasserstoff 207

8. Reaktion: Ersatz der Diazogruppe durch Hydroxyl . . . . . . 213

9. Reaktion: Ersatz einer Diazogruppe durch Jod . . . . . . . 215

10. Reaktion: Ersatz einer Diazogruppe durch Chlor, Brom oder Cyan 218

11. Reaktion: a) Reduktion einer Diazoverbindung zu einem Hydrazin. b) Ersatz des Hydrazinrestes durch Wasserstoff . . . . . . 220

12. Reaktion: a) Darstellung eines Azofarbstoffes aus einer Diazoverbindung und einem Amin. b) Reduktion desselben . . . . . 226

13. Reaktion: Darstellung eines Diazoamidokörpers . . . . . . . 231

14. Reaktion: Umlagerung eines Diazoamidokörpers in einen Amidoazokörper . . . . . . . . . . . . . . . . . . . . 233

15. Reaktion: Oxydation eines Amins zu einem Chinon . . . . . 234

16. Reaktion: Reduktion eines Chinons zu einem Hydrochinon . . . 238

17. Reaktion: Bromierung eines aromatischen Körpers . . . . . . 239 
18. Reaktion: Fitrigs Synthese eines Kohlenwasserstoffs . . . . . 244

19. Reaktion: Sulfurierung eines aromatischen Kohlenwasserstoffs (I) - 246

20. Reaktion: Reduktion eines Sulfochlorids zu einer Sulfinsäure oder zu einem 'Thiophenol . . . . . . . . . . . . . . . . 254

21. Reaktion: Sulfurierung eines aromatischen Kohlenwasserstoffs (II) 257

22. Reaktion: Überführung einer Sulfosüure in ein Phenol . . . . 259

23. Reaktion: Nitrierung eines Phenols . . . . . . . . . . . 261

24. Reaktion: a) Chlorierung eines Kohlenwasserstoffs in der Seitenkette. b) Überführung eines Dichlorids in einen Aldehyd . . 263

25. Reaktion: Gleichzeitige Oxydation und Reduktion eines Aldehyds unter dem Einflusse von konzentriertem Kali . . . . . . . 269

26. Reaktion: Kondensation eines Aldehyds durch Cyankalium zu einem Benzoin . . . . . . . . . . . . . . . . . . . . . 270

27. Reaktion: Oxydation eines Benzoins zu einem Benzil . . . . . 272

28. Reaktion: Anlagerung von Cyanwasserstoff an einen Aldehyd . 273

29. Reaktion: Perkis sche Zimtsăuresynthese . . . . . . . . . 278

30. Reaktion: Anlagerung von Wasserstoff an ein Äthylenderivat . . 281

31. Reaktion: Darstellung eines aromatischen Säurechlorids aus der Säure und $\mathrm{PCl}_{5}$. . . . . . . . . . . . . . . . . . 281

32. Reaktion: Schotтen-Baumannsche Reaktion zur Erkennung $\mathbf{N H}_{\mathbf{2}}$ bezw. NH- bezw. OH-Gruppen enthaltender Verbindungen . . 283

33. Reaktion: a) Ketonsynthese nach Frieder. und Crafts. b) Darstellung eines Oxims. c) BвскMaNnsche Umlagerung eines Oxims

34. Reaktion: Reduktion eines Ketons zu einem Koblenwasserstoff . 293

35. Reaktion: Aldehydsynthese nach Gattermann-Kocı . . . . . . 294

36. Reaktion: Verseifung cines Säurenitrils . . . . . . . . . . 298

37. Reaktion: Oxydation der Seitenkette eines aromatischen Körpers . 300

38. Reaktion: Synthese von Oxyaldehyden nach REIMER und TiEmanN 303

39. Reaktion: Kolbes Synthese von Oxysäuren . . . . . . . . . 307

40. Reaktion: Grignards Reaktion. a) Benzoesăure aus Jodbenzol. b) Benzhydrol aus Jod- oder Brombenzol und Benzaldehyd . . 310

41. Reaktion: Darstellung eines Farbstoffs der Malachitgrünreihe . . 316

42. Reaktion: Kondensation von Phtalsăureanhydrid mit einem Phenol zu einem Phtalein. . . . . . . . . . . . . . . . . 319

43. Reaktion: Kondensation von Michlerschem Keton mit einem Amin zu einem Farbstoff der Fuchsinreihe . . . . . . . . . . 325

44. Reaktion: Kondensation von Phtalsäureanhydrid mit einem Phenol zu einem Anthrachinonderivat . . . . . . . . . . . . 326

45. Reaktion: Alizarin aus $\beta$-anthrachinonmonosulfosaurem Natrium . 328

46. Reaktion: Zinkstaubdestillation . . . . . . . . . . . . . 330

III. Pyridin- und Chinolinreihe.

1. Reaktion: Pyridinsynthese von $\mathrm{H}_{\Delta \mathrm{NTzs}}$. . . . . . . . . 332

2. Reaktion: Skratpsche Chinolinsynthese . . . . . . . . . . 335 
IV. Anorganiseher Teil.

1. Chlor $\ldots 337$

2. Chlorwasserstoff. . . . . . . . . . . . . . . . . . . 338

3. Bromwasserstoff . . . . . . . . . . . . . . . . . . 339

4. Jodwasserstoff . . . . . . . . . . . . . . . . . . . 340

5. Ammoniak . . . . . . . . . . . . . . . . . . . . 342

6. Salpetrige Säure . . . . . . . . . . . . . . . . . . 342

7. Phosphortrichlorid . . . . . . . . . . . . . . . . . . 342

8. Phosphoroxychlorid . . . . . . . . . . . . . . . . . 343

9. Phosphorpentachlorid . . . . . . . . . . . . . . . . . 344

10. Schweflige Säure . . . . . . . . . . . . . . . . . . 344

11. Natrium . . . . . . . . . . . . . . . . . . . . . 345

12. Aluminiumchlorid . . . . . . . . . . . . . . . . . . 346

13. Bleisuperoxyd . . . . . . . . . . . . . . . . . . . 348

14. Kupferchlorür . . . . . . . . . . . . . . . . . . . . . 349

15. Wertbestimmung des Zinkstaubs . . . . . . . . . . . . 349

Register . . . . . . . . . . . . . . . . . . . 351

Trbelle zur Berechnung der Stickstoff-Bestimmungen.

\section{Abkürzungen.}

A. = Liebras Annalen der Chemie.

A. ch. = Annales de chimie et de physique.

B. $=$ Berliner Berichte.

Bl. = Bulletin de la société chimique de Paris.

Ch.-Z. = Chemiker-Zeitung.

J. = Jahresbericht über die Fortschritte der Chemie.

J. pr. = Journal für praktische Chemie.

P. = Pogozndorprs Annalen.

R. = Journal der russischen chemischen Gesellschaft.

Z. = Zeitschrift für Chemie. 